\title{
Wideband Negative Permittivity and Double Negative Fishnet-Mushroom-Like Metamaterial in X-Band Waveguide
}

\author{
Ahmed Mahmood, ${ }^{1}$ Golge Ogucu Yetkin, ${ }^{1}$ and Cumali Sabah ${ }^{2,3}$ \\ ${ }^{1}$ Department of Electrical and Electronics Engineering, University of Gaziantep, 27310 Gaziantep, Turkey \\ ${ }^{2}$ Department of Electrical and Electronics Engineering, Middle East Technical University-Northern Cyprus Campus (METU-NCC), \\ Kalkanli, Guzelyurt, 99738 TRNC/Mersin 10, Turkey \\ ${ }^{3}$ Kalkanli Technology Valley, Middle East Technical University-Northern Cyprus Campus (METU-NCC), Kalkanli, Guzelyurt, \\ 99738 TRNC/Mersin 10, Turkey \\ Correspondence should be addressed to Cumali Sabah; sabah@metu.edu.tr
}

Received 21 November 2016; Revised 21 January 2017; Accepted 31 January 2017; Published 28 February 2017

Academic Editor: Charles Rosenblatt

Copyright (C) 2017 Ahmed Mahmood et al. This is an open access article distributed under the Creative Commons Attribution License, which permits unrestricted use, distribution, and reproduction in any medium, provided the original work is properly cited.

\begin{abstract}
A Fishnet-Mushroom-like metamaterial electromagnetic behaviour is represented in $S$-parameters numerically and experimentally for X-band frequencies arena. The design has introduced a dielectric substrate as a host with metallic parts. The proposed design is predicted to provide the electromagnetic band gap characterization with desired reiterative characteristic parameters, negative permittivity, and negative permeability exhibiting a double negative left-handed region, which is identified with the X-band regime with good agreement between the simulated and the measured results.
\end{abstract}

\section{Introduction}

Over the past decade, interest in applications metamaterials (MTMs) has grown explosively; metamaterials are a kind of a material with electromagnetic properties, which are not readily available in nature. Under certain conditions, these materials provide a simultaneous negative permittivity $(-\varepsilon)$ and permeability $(-\mu)$ in certain frequency range, and they exhibit reversal of Snell's Law (negative refractive index) and Doppler Effect and Cerenkov Effect around the transmission peak. Depending on the negative value of the permittivity (real part), permeability (real part), or both, metamaterials can be categorized as epsilon negative (ENG), mu negative (MNG), and/or double negative (DNG), which presented the left-handed metamaterials (LHM) they were first proposed by Veselago [1]. Conventional materials have permittivity and also permeability larger than zero in all frequency ranges and they are called double positive (DPS). They become very desirable for designing and fabricating MTMs that can achieve these properties, according to their possible applications in diverse engineering and scientific fields. The metamaterials are designed to use dielectric as host and metal containment, which have been fabricated and then tested intensively in the form of different periodical arrangement [2, 3]. Recently, the fishnet structure has attracted a great potential approved in the studies where the negative refraction index for first time was displayed in a 3D optical MTM $[4,5]$. Basically, the strategy to acquire the negative permeability is to create exciting circular currents that can generate a strong magnetic resonance, while being expected to produce the negative permittivity by virtue of the electric plasma response with added continuous wires. When negative refraction index is inconsistent with the negative permeability, most of fishnet structure researches concentrated on characterizing and modeling their magnetic resonance [6-9].

The dispersion diagram of a $2 \mathrm{D}$-periodic structure is produced by calculating the resonant frequencies of Eigen modes, which present differential equation solutions that simulate the fields in a single MTM cell. However, a different band gap acquired by a restricted amount of surface waves along the contour of the irreducible Brillouin zone for an infinite array of the proposed MTM structure will be 


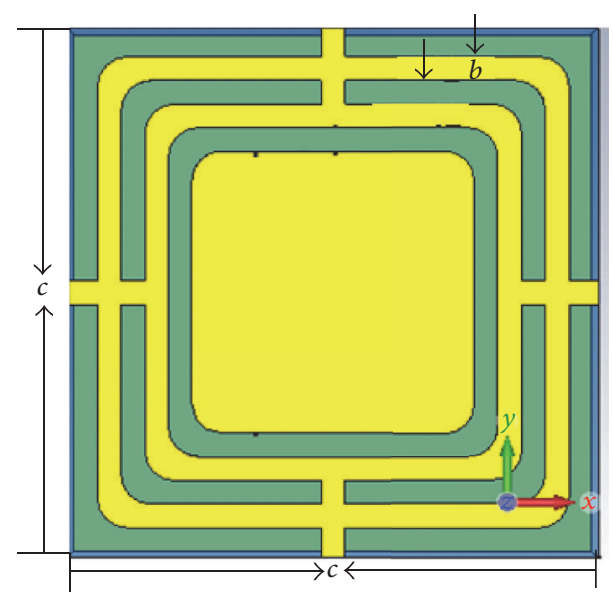

(a)

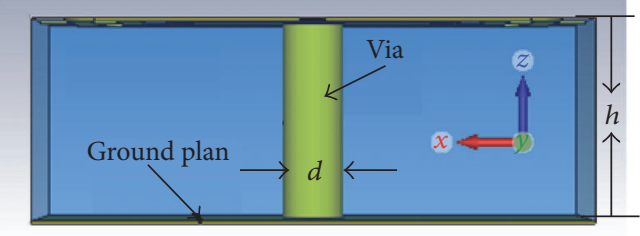

(b)

Figure 1: The EBG unit cell schematic. (a) Top view. (b) Side view.

produced depending on how many metamaterials arrays are presented. Therefore, only the dispersion relation can obtain the exact position of passband and band gap in the frequency spectrum.

The study presents two cases of MTMs. The first one is a wideband $(-\epsilon)$ negative permittivity (ENG), whereas the second one is double negative (DNG). Both cases are the electromagnetic behaviour represented in $S$-parameters that are numerically and experimentally studied. The simulated and the experimental results exhibit very good agreement in the (8-12) GHz X-band regime whereas the permittivity and permeability and refraction index characteristics are extracted to explain the behaviour of the MTM cell. The proposed MTM (Fishnet-Mushroom-like) is fabricated and measured. The obtained results are compared with simulated results, which are carried out by CSTMWs' simulation program [10]. The given notes, as well as the NicolsonRoss-Weir (NRW) procedure, are considered to obtain the properties of the designed MTM structure and the effective material parameters $[11,12]$.

\section{Features of Electromagnetic Band Gap (EBG) Structure}

2.1. Unit Cell Design. The proposed metamaterial EBG unit cell consisted of a square substrate with $4.5 \mathrm{~mm}$ side length and $1.6 \mathrm{~mm}$ thickness. The FR-4 epoxy with a permittivity $\varepsilon_{r}=4.3$ and loss tangent $\delta=(0.025)$ is chosen to represent the substrate material. The metal shape is printed on the substrate front side which is composed of a squared patch surrounded by two square rings connected from their center sides like a net. A cylindrical via with a diameter of $0.5 \mathrm{~mm}$ is also located in the unit cell center as shown in Figure 1(b), by which the square patch and the ground plan in the bottom side are connected. Copper of electrical conductivity $\sigma=$ $5.8 \times 10^{7} \mathrm{~S} / \mathrm{m}$ with coating thickness $0.017 \mathrm{~mm}$ is chosen for printing as shown in Figure 1(a). the unit cell dimension parameters are mentioned in Table 1.
TABLE 1: Structure dimensions for the EBG unit cell.

\begin{tabular}{lc}
\hline Parameter & $\mathrm{mm}$ \\
\hline$a$ & 2.4 \\
$b$ & 0.2 \\
$c$ & 4.5 \\
$d$ & 0.5 \\
$h$ & 1.6 \\
\hline
\end{tabular}

2.2. Dispersion Diagram. The concept of this paper states that the wave propagation direction is assumed in the same direction of the unit cells periodicity. The space harmonics share the same group velocity even if they have different phase velocities for they cannot occur separately. Each single harmonic does not satisfy the boundary conditions of the periodic structure, but their summation does so. Thus, summation is considered to be the same mode. Hence, by simulating a single proposed EBG unit cell structure, an infinite periodic structure model is carried out by CSTMWs eigenmode simulator.

The band gap characterization for EBG is shown in Figure 2. We expound the idea that the field for the first mode is TM dominant, started at zero frequency, and the eigenfrequency increases with the wave number, reaching the maximum frequency $8.45 \mathrm{GHz}$. It also decreases following the light line down to a certain frequency. The second mode starts at a higher frequency and continues increasing with a slope under the vacuum's speed of light, which is set by a straight line. The band gap is near $4 \mathrm{GHz}$ for this particular structure (8.45-13.15 GHz), comparing to previous structure designs that achieved less band gap in the same arena $[13,14]$.

2.3. Array Structure Design and Simulations Settings. The simulations included the proposed unit cell and are ordered by varying different arrays arrangements [15], like $1 \times 8$ cell array and $3 \times 8$-, $4 \times 8$-, and $6 \times 8$-cell arrays. These 


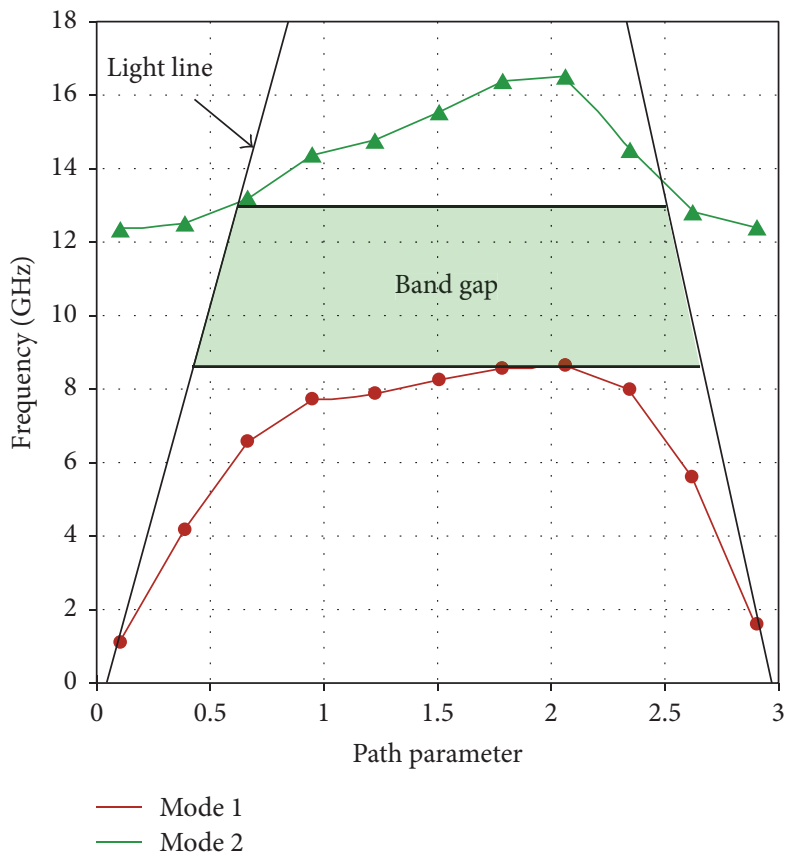

FIgURE 2: Dispersion diagram.

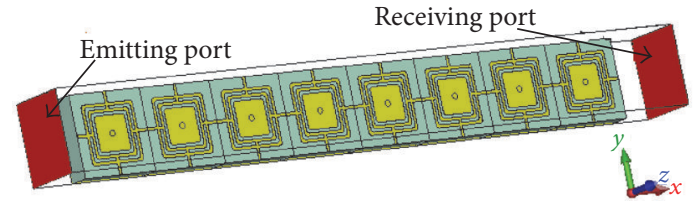

(a)

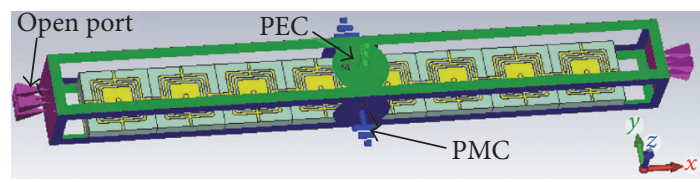

(b)

FIGURE 3: EBG array simulation settings achieved the band gap measurements. (a) EBG characterization principle; (b) waveguide boundary conditions.

arrangements are set between two waveguide ports in the $x$ directions in order to simulate $S$-parameters and obtain the reiterative characteristic parameters, and the band gap effect of varying the amount of metamaterial is so.

The EBG is analyzed and simulated by applying the sample to a surface wave propagation measurement through the setting procedures that presented in (Figures 3(a) and $3(\mathrm{~b}))$.

Furthermore, microwave ports are set on the $x$-axis sides, on which one port is located as a transmitter generating electromagnetic wave $\left(E^{\rightarrow}, H^{\rightarrow}\right)$ and the second port as a receiver. These boundary conditions are chosen in order to define a waveguide structure (Figure 3(b)). Moreover, a perfect magnetic boundary condition (PMC) is set in the $y$ directions in which infinite periodic repeating in the same directions for the eight EBG and a perfect electric boundary condition (PEC) are set in the $z$-directions, considering a ground plane placed in the below side of the EBG structure and with the open boundaries in the $x$-directions where the ports are set.

\section{Results and Discussion}

For an experimental reason to suit with the WR-90 dimensions and to decrease the misalignment experiment duration such as position of the fabricated sample plate, the array of $4 \times 8$ arrangement is chosen and fabricated as shown in Figure 4(a), whereas Figure 4(b) showed the schematic experiment.

The reflection coefficient $S_{11}$, transmission coefficient $S_{21}$, and the reiterative characteristic parameters have a negative index, whereas the permittivity and the permeability are in the $\mathrm{X}$-band frequency range. The simulation results exhibit that the structure has been designed successfully; that is, the simulation and experimental data agree with each other as possible.

The experiment steps are as follows: First, the WR-90 waveguide is connected with the input and the output ports with the network analyzer by the two coaxial probes, and the network analyzer is calibrated. Second, the fabricated sample is fixed at the center of a WR-90 waveguide; all the metallic part that is used is unconnected to the waveguide inner walls. The dielectric substrate thickness has chosen being as thin as possible to minimize the dielectric loss. The selected structure dimensions which severed the optimization goal had proven the double negative performance at around X-band arena $[11,12]$. 

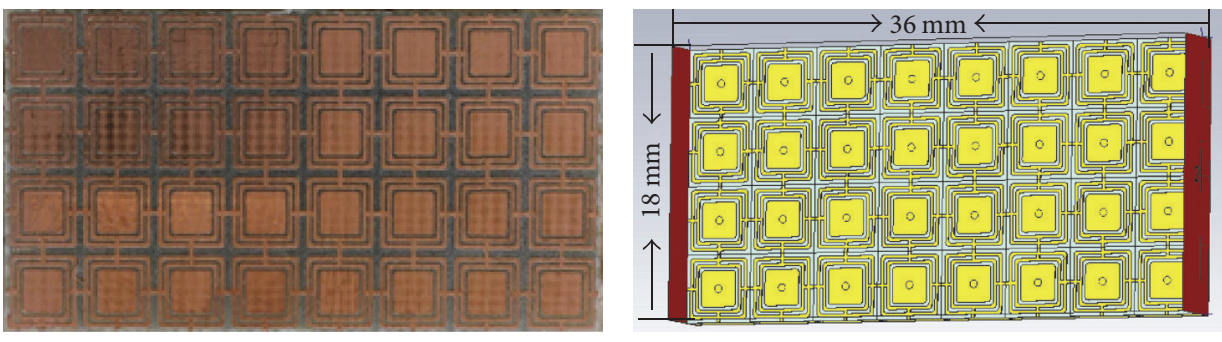

(a)

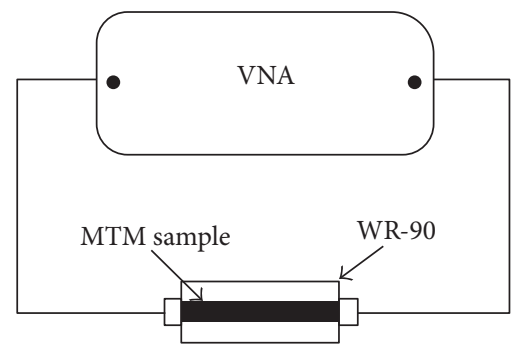

(b)

Figure 4: (a) The fabricated $4 \times 8$ arrangement sample; (b) experimental set-up schematic representation. VAN: vector network analyzer.

Referring to the method described in Section 2.2, surface waves propagating and travelling through the circuit board are detected and the amplitude and phase for the reflection and transmission data measured. Figure 5(a) shows both simulated and measured $S$-parameter data for the investigated structure in good agreement between them.

The experimental and the simulated results show a small difference in minor deviations between the measured and the simulated data that are pointed to manufacture allowance, which is related to PCB fabrication and the gathering process and the quality of the dielectric FR4 dispersion used as a substrate. Likewise, the misalignment experiment duration such as position of the fabricated sample plate, which cannot be neglected, port receptacle locations, and loss characteristics of the materials are regarded as another root of error. On the other hand, the extent of measurement accuracy can be elucidated through the convergence between the results of experimental and simulations outcome.

The effective parameters for the obtained investigated structure material are based on the effectively homogeneous medium occurring when the periodicity of the structure is much less than the propagation wavelength in the propagation direction. The experimented metamaterial is treated as a LHM in the region between 10.95 and $11 \mathrm{GHz}$, and the transmission peak is indicated at $10.975 \mathrm{GHz}$. It is clearly stated that the boundary conditions selected to consider the designed structure as an infinite homogeneous material in a certain region led to a frequency observation [14], where both the dielectric permittivity and magnetic permeability are simultaneously negative, which resulted in the retrieval method application.

The NRW method is used in the material parameters calculation extracted and presented in Figures 5(a) and 5(b). As the figure shows, the metamaterial structure exhibits a negative permittivity in all X-band simultaneously with negative permeability in a band between 10.950 and $11 \mathrm{GHz}$, which a high band transmission peak covered. In spite of the negative refraction index, wideband included all the X-band (see Figure 5(b)), and that type of the negative refraction index is not supporting the left-hand (LH) behaviour. As it was previously mentioned, the representation of the single negative (SNG) occurred only with negative real part of the permittivity. Consequently, both the single negative (SNG) (between 8 and $10.95 \mathrm{GHz}$ and between 11 and $12 \mathrm{GHz}$ ) and the double negative (DNG) (10.95-11 GHz) $\mathrm{LH}$ region characteristics have been supported by the real part of the negative refractive index $[16,17]$. Due to the electric field and the copper parts conjunction and because of strips as in the continuous-wires case, the permittivity exhibits a Drude-like behaviour. Also, the permeability shows Lorentz-like behaviour, through a strong resonant response to the magnetic field during the magnetic resonators condition.

Figures 6(a) and 6(b) show the simulated propagation of surface waves and currents due to the presence of EBG $4 \times 8$ structure. It is exhibited from these simulations, when surface currents are generated from (port 1 ) side in $x$-direction which then travels through the EBG structure opposite side (port 2). In Figure 6(a), the EBG structure with an operating frequency band - the single negative (SNG) (between 8 and $10.95 \mathrm{GHz}$ and between 11 and $12 \mathrm{GHz}$ ) - was applied. As expected, the band gap effect shown in Figure 2 does not support any wave propagation at a frequency included within its band gap and suffering reduction. On the other hand, Figure 6(b) demonstrates the LHM in the region between 10.95 and $11 \mathrm{GHz}$ and as a consequence of LHM behaviour the EBG structure surface presence results in a symmetric distribution of the surface current from port 1 to port 2. 

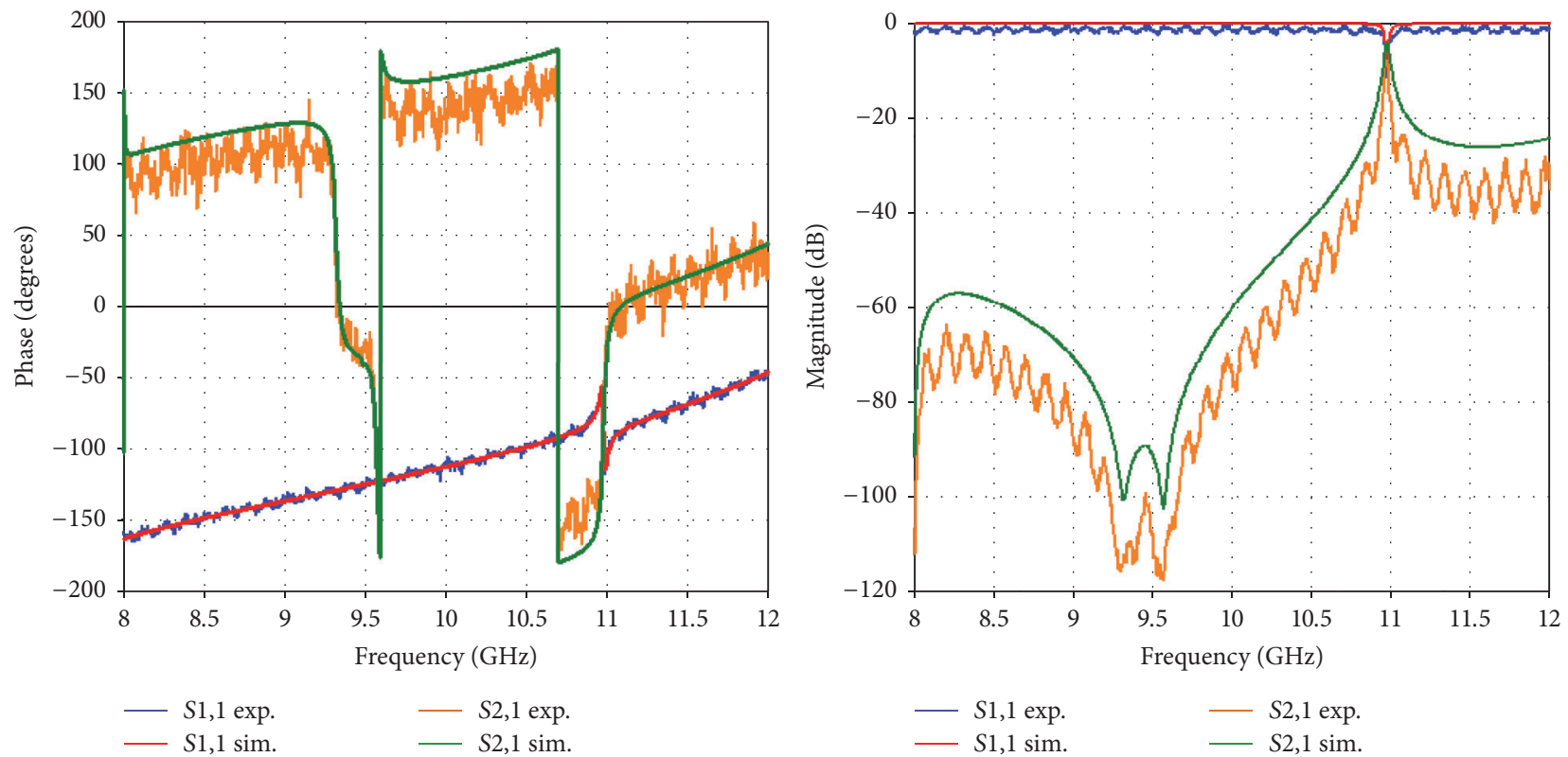

(a)

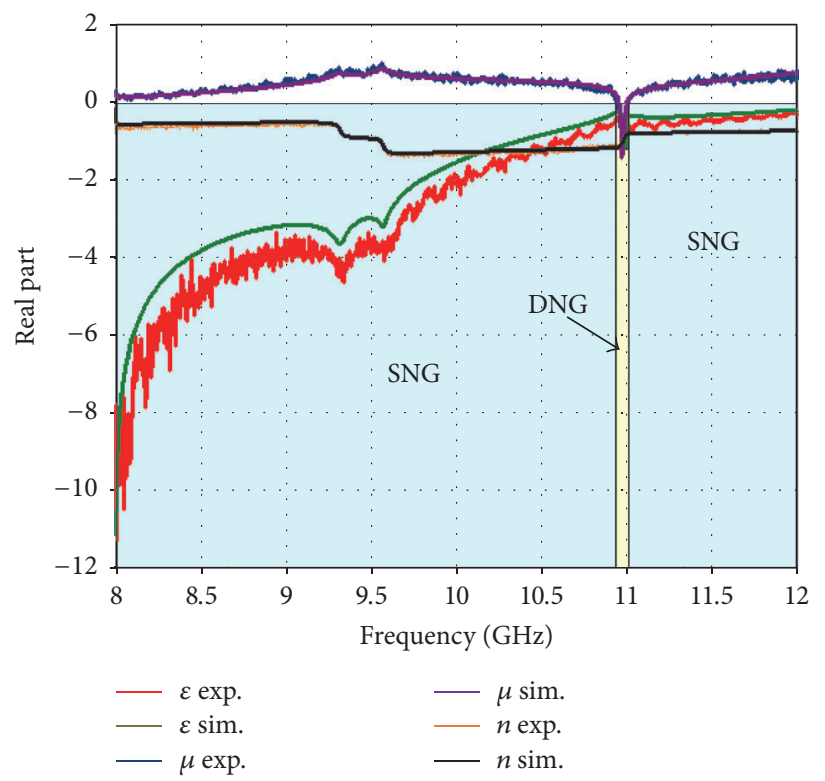

(b)

Figure 5: Comparison between simulation and measured results. (a) The Fishnet-Mushroom-like MTMs' S-parameters. (b) Retrieved characteristic parameters.

\section{Conclusion}

In this study, the proposed Fishnet-Mushroom-like MTM is fabricated and measured, and the obtained results are compared with simulated results. There are two cases of MTMs, the first one of which is $(-\epsilon)$ a wideband negative permittivity (between 8 and $10.95 \mathrm{GHz}$ and between 11 and $12 \mathrm{GHz}$ ) and the second one is double negative (DNG) (between 10.95 and $11 \mathrm{GHz}, \mathrm{LH}$ region; and $10.975 \mathrm{GHz}$ is indicated as transmission peak). The electromagnetic behaviour for the said cases in question is represented in $S$-parameters numerically and experimentally for the X-band arena. The value of this study brings forth the fact that this structure can be used in wideband (ENG) applications such as efficient electrically small antenna enhancement applications and also for double-negativity antenna and radar cross section (RCS) property enhancement applications [18-21].

\section{Competing Interests}

The authors declare that there is no conflict of interests regarding the publication of this paper. 

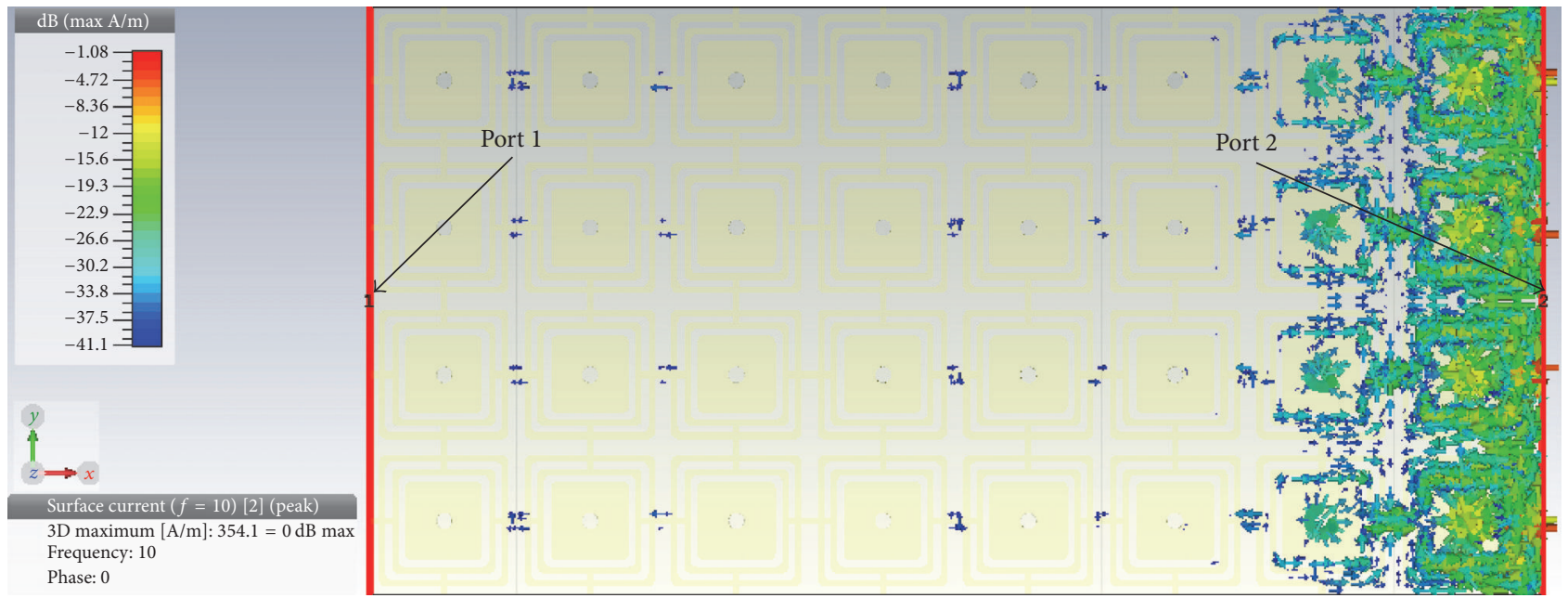

(a)
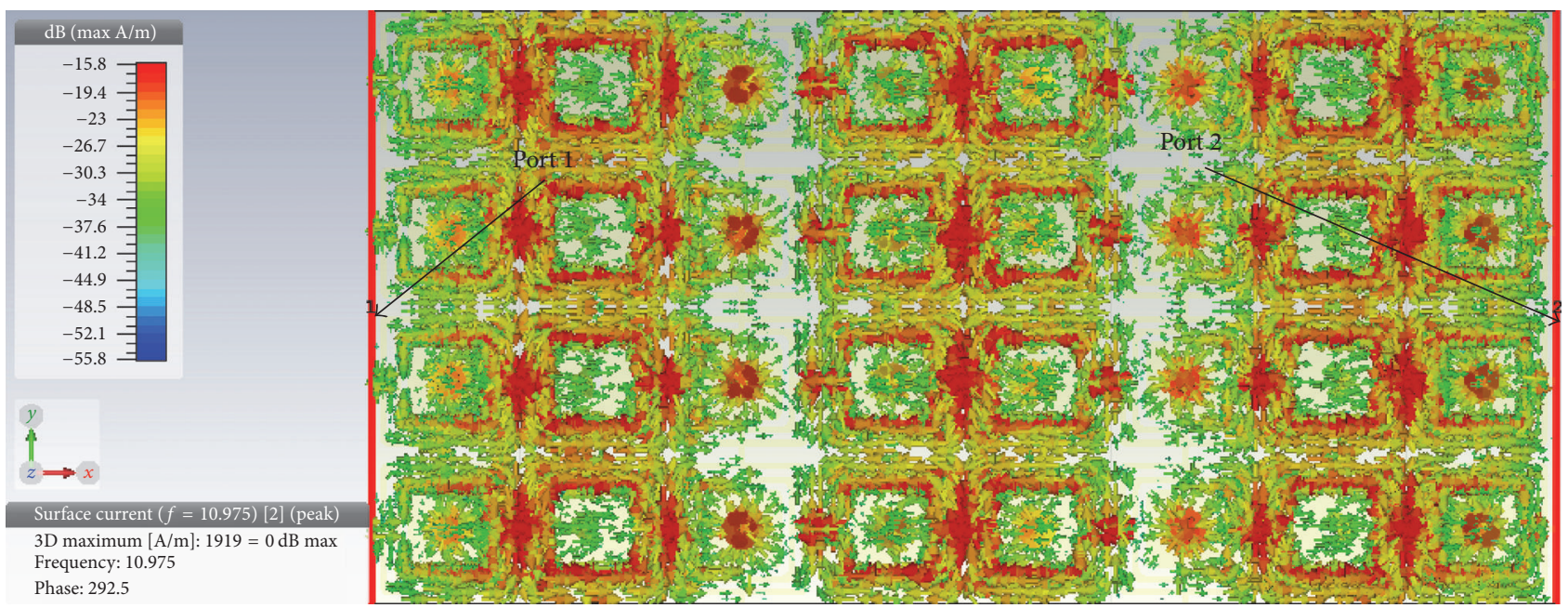

(b)

FIGURE 6: (a) Showing the simulated propagation of surface currents for $10 \mathrm{GHz}$ in the SNG region. (b) Showing the simulated propagation of surface currents for $9.975 \mathrm{GHz}$ in the DNG (LHM) region.

\section{References}

[1] V. G. Veselago, "The electrodynamics of substances with simultaneously negative values of $\varepsilon$, and $\mu$,' Soviet Physics Uspekhi, vol. 10, no. 4, pp. 509-514, 1968.

[2] A. I. Mackenzie, "Comparison of two AMC's on a highpermittivity substrate," in Proceedings of the 31st International Review of Progress in Applied Computational Electromagnetics (ACES '15), March 2015.

[3] A. I. Mackenzie, "Microwave band gaps produced by varying numbers of mushroom metamaterial cells," in Proceedings of the IEEE Antennas and Propagation Society International Symposium (APS '15), pp. 1102-1103, IEEE, Vancouver, BC, Canada, July 2015.

[4] J. Valentine, S. Zhang, T. Zentgraf et al., "Three-dimensional optical metamaterial with a negative refractive index," Nature, vol. 455, pp. 376-379, 2008.

[5] M. Kafesaki, I. Tsiapa, N. Katsarakis, Th. Koschny, C. M. Soukoulis, and E. N. Economou, "Left-handed metamaterials: the fishnet structure and its variations," Physical Review B, vol. 75, no. 23, Article ID 235114, 2007.

[6] V. D. Lam, J. B. Kim, S. J. Lee, and Y. P. Lee, "Left-handed behavior of combined and fishnet structures," Journal of Applied Physics, vol. 103, no. 3, Article ID 033107, 2008.

[7] A. Koray, L. Zhaofeng, S. Levent, and O. Ekmel, "Negative phase advance in polarization independent, multi-layer negativeindex metamaterials," Optics Express, vol. 16, no. 12, pp. 88358844, 2008.

[8] N. T. Tung, V. D. Lam, M. H. Cho, J. W. Park, W. H. Jang, and Y. P. Lee, "Influence of the dielectric-spacer thickness on the lefthanded behavior of fishnet metamaterial structure," Photonics and Nanostructures-Fundamentals and Applications, vol. 7, no. 4, pp. 206-211, 2009.

[9] CST Microwave Studios 2016, http://www.cst.com/.

[10] R. Ahmadian, S. Sharma, M. Rahimi, and F. B. Zarrabi, "Investigation of EBG array performance on decreasing the mutual coupling," in Proceedings of the 4th International Conference on 
Advanced Computing and Communication Technologies (ACCT '14), pp. 359-362, February 2014.

[11] S. Sahandabadi, F. H. Kashani, and M. Fallah, "Sierpinski triangular antenna on a mushroom-like EBG metamaterial ground plane," Journal of Telecommunication, vol. 23, no. 2, 2014.

[12] V. S. Aravind and S. Gupta, "Compact EBG ground plane microstrip antenna for high gain applications," in Proceedings of the 2nd International Conference on Emerging Technology Trends in Electronics, Communication and Networking (ET2ECN '14), pp. 1-3, IEEE, Surat, India, December 2014.

[13] S. S. Islam, M. R. I. Faruque, and M. T. Islam, "The design and analysis of a novel split-H-shaped metamaterial for multi-band microwave applications," Materials, vol. 7, no. 7, pp. 4994-5011, 2014.

[14] C. Sabah, A. O. Cakmak, E. Ozbay, and S. Uckun, "Transmission measurements of a new metamaterial sample with negative refraction index," Physica B: Condensed Matter, vol. 405, no. 14, pp. 2955-2958, 2010.

[15] C. Sabah, "Realization of polarization-angle-independent fishnet-based waveguide metamaterial comprised of octagon shaped resonators with sensor and absorber applications," Journal of Materials Science: Materials in Electronics, vol. 275, pp. 4777-4787, 2016.

[16] N. T. Tung, V. D. Lam, J. W. Park et al., "Single- and doublenegative refractive indices of combined metamaterial structure," Journal of Applied Physics, vol. 106, Article ID 053109, 2009.

[17] C. Sabah and H. G. Roskos, "Numerical and experimental investigation of fishnet-based metamaterial in a X-band waveguide," Journal of Physics D: Applied Physics, vol. 44, no. 25, Article ID 255101, 2011.

[18] Q.-R. Zheng, Y.-M. Yan, X.-Y. Cao, and N.-C. Yuan, "High impedance ground plane (HIGP) incorporated with resistance for radar cross section (RCS) reduction of antenna," Progress in Electromagnetics Research, vol. 84, pp. 307-319, 2008.

[19] G. Singh, R. ni, and A. Marwaha, "A review of metamaterials and its applications," International Journal of Engineering Trends and Technology, vol. 19, no. 6, pp. 305-310, 2015.

[20] Q. Tang, B. Chen, W. Luo, and P. Tang, "Design of a substrate integrated waveguide aperture antenna with electromagnetic band gap structure," in Proceedings of the IEEE International Conference on Communication Problem-Solving (ICCP '15), Guilin, China, October 2015.

[21] Y. Rahmat-Samii and H. Rajagopalan, "From a PEC ground plane to an EBG surface: understanding the underlying physics," in Proceedings of the International Symposium on Antennas and Propagation (ISAP '09), Bangkok, Thailand, October 2009. 

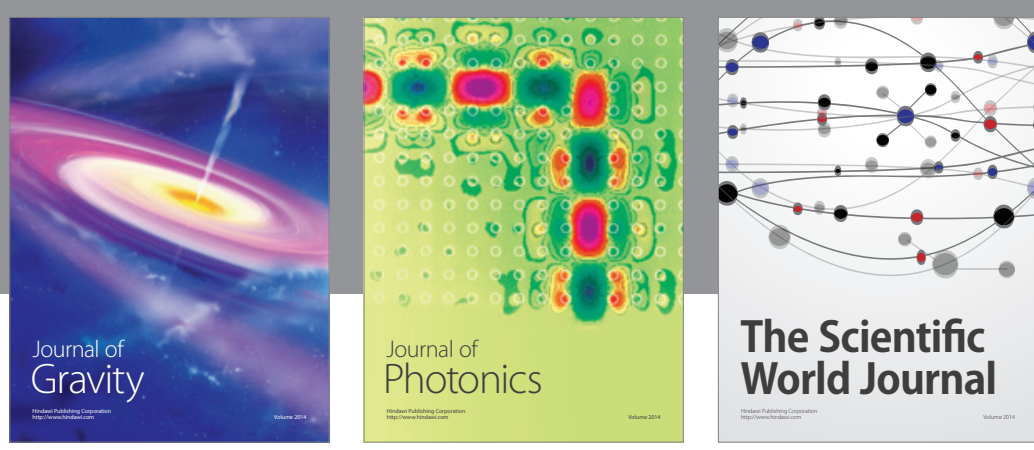

The Scientific World Journal
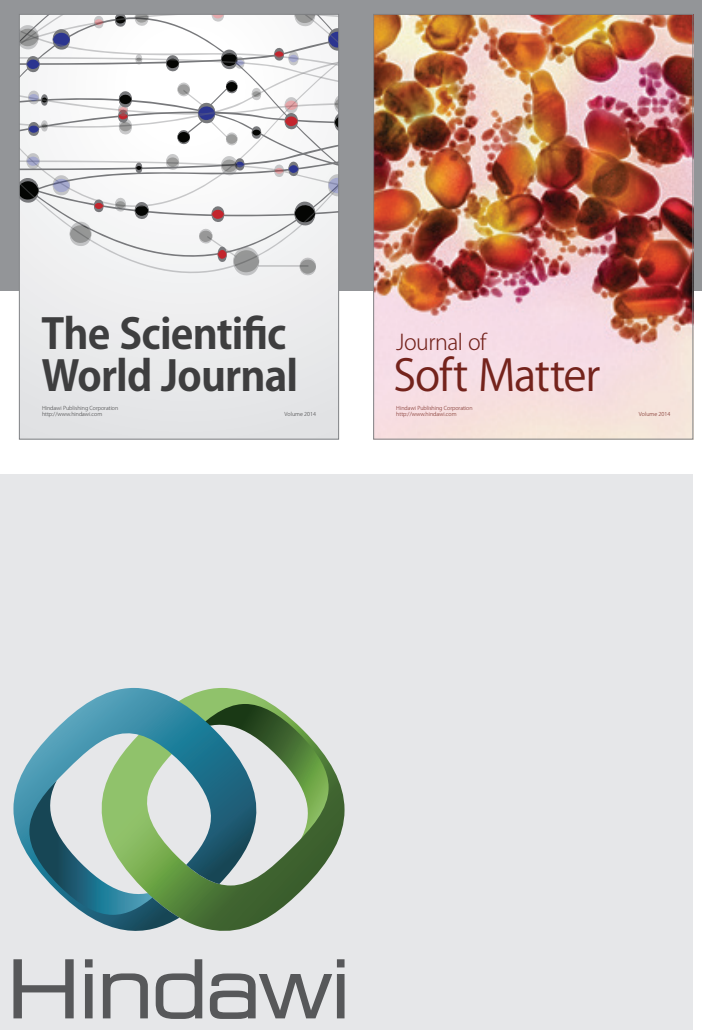

Submit your manuscripts at

https://www.hindawi.com
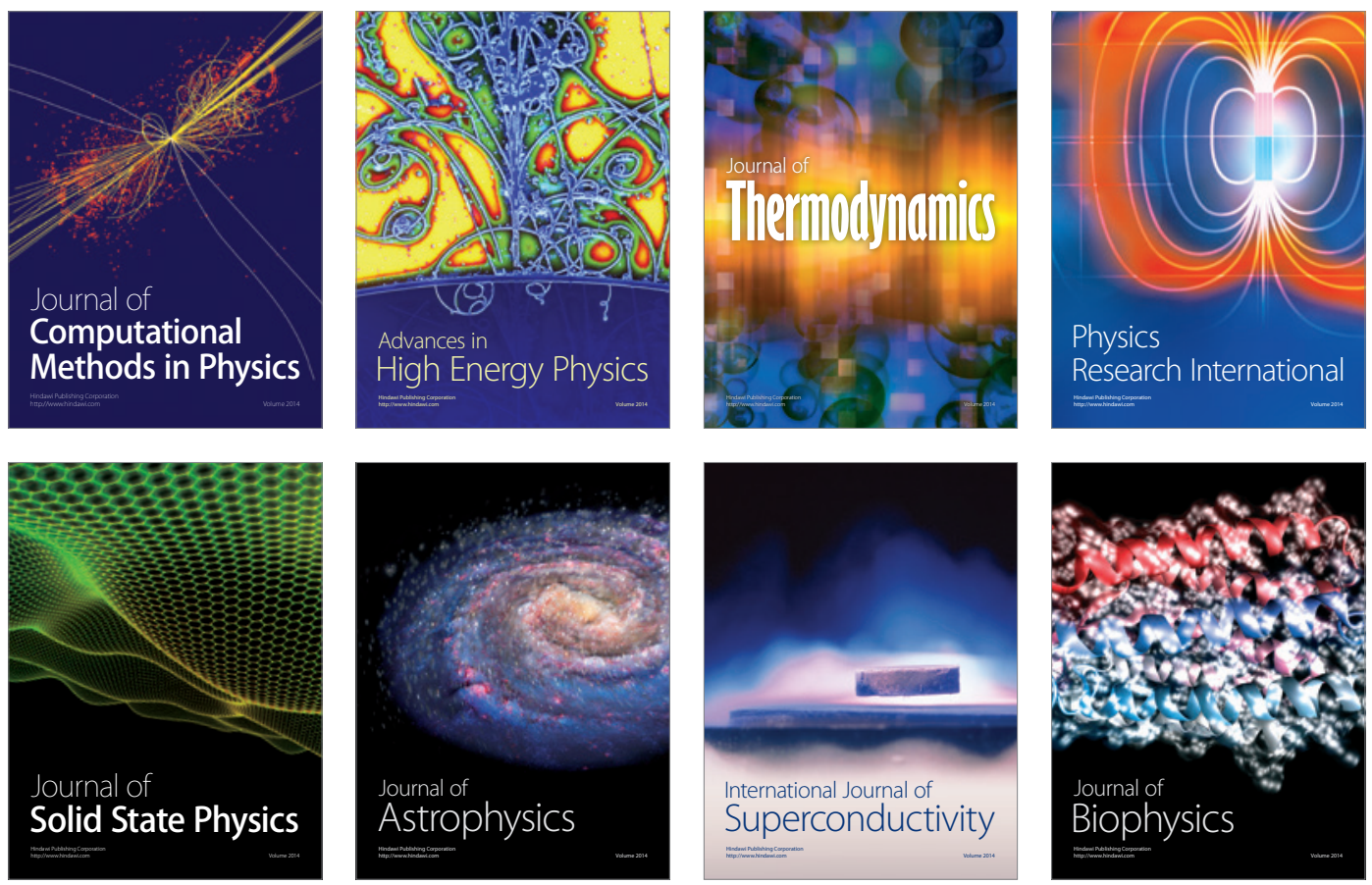
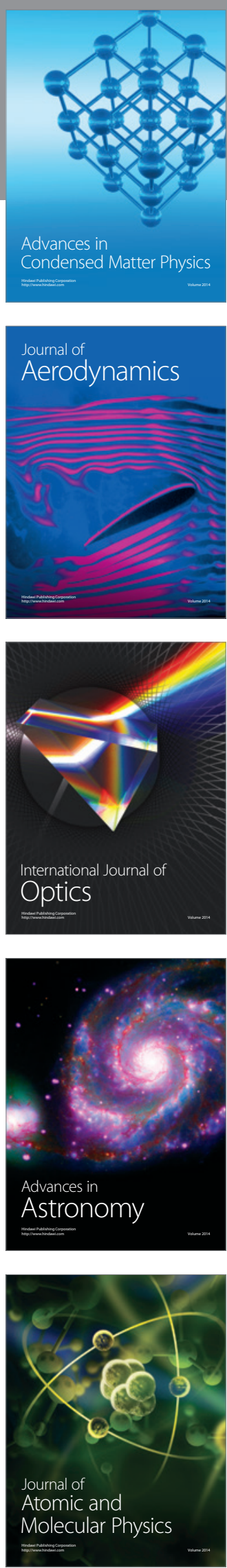